\title{
Validation of Diagnostic Markers for Streak Virus Disease Resistance in Maize
}

\author{
Solomon Shibeshi Sime ${ }^{1,2}$, Abebe Menkir ${ }^{1}$, Victor O. Adetimirin ${ }^{3}$, Melaku Gedil ${ }^{1}$ (D) and P. Lava Kumar ${ }^{1, * \mathbb{D}}$ \\ 1 International Institute of Tropical Agriculture (IITA), Oyo Road, Ibadan PMB 5320, Nigeria; \\ shibeshisolomon23@gmail.com (S.S.S.); a.menkir@CGIAR.ORG (A.M.); M.Gedil@cgiar.org (M.G.) \\ 2 Pan African University Life and Earth Science Institute, University of Ibadan, Ibadan 200284, Nigeria \\ 3 Department of Crop and Horticultural Sciences, University of Ibadan, Ibadan 200284, Nigeria; \\ votimirin@yahoo.com \\ * Correspondence: L.kumar@cgiar.org; Tel.: +234-7032-565-130
}

Citation: Sime, S.S.; Menkir, A.; Adetimirin, V.O.; Gedil, M.; Kumar, P.L. Validation of Diagnostic Markers for Streak Virus Disease Resistance in Maize. Agriculture 2021, 11, 130. https://doi.org/10.3390/ agriculture11020130

Academic Editor: Manuel Jamilena Received: 30 December 2020

Accepted: 1 February 2021

Published: 5 February 2021

Publisher's Note: MDPI stays neutral with regard to jurisdictional claims in published maps and institutional affiliations.

Copyright: (C) 2021 by the authors. Licensee MDPI, Basel, Switzerland. This article is an open access article distributed under the terms and conditions of the Creative Commons Attribution (CC BY) license (https:// creativecommons.org/licenses/by/ $4.0 /)$.

\begin{abstract}
Maize streak virus (MSV) is responsible for streak disease of maize and poses a serious threat to maize production in sub-Saharan Africa. Polygenic resistance to MSV has become an essential requirement in modern maize cultivars to mitigate yield losses. Many single nucleotide polymorphism (SNP) markers linked to putative MSV resistance loci have been identified for use in forward breeding. This study aimed to validate, using the high-throughput kompetitive allele specific PCR (KASP) assay, the diagnostic ability of the three SNP markers linked to the loci for the Msv1 resistance trait in 151 early generations inbred lines with diverse genetic backgrounds, together with nine MSV-resistant elite lines and a susceptible check (cv. Pool-16). The phenotypic responses were determined by MSV inoculation using viruliferous leafhoppers (Cicadulina triangular) under screenhouse conditions. Based on an established MSV disease rating system, the maize lines were categorized into resistant, moderately resistant, susceptible, and highly susceptible. The three SNPs associated with MSV resistance were detected in 133 lines, which were categorized as resistant (54), moderately resistant (76), and susceptible (1). The 18 early generation lines without these SNPs were classified as moderately resistant (10), susceptible (5), and highly susceptible (3). This study confirms the strong association of SNPs with MSV resistance and their usefulness for forward breeding in maize while emphasizing the need to identify additional markers to screen lines for MSV resistance without any ambiguity.
\end{abstract}

Keywords: maize; maize streak virus; phenotyping; genotyping; quantitative resistance; markerassisted breeding

\section{Introduction}

Maize streak disease (MSD) is caused by Maize streak virus (MSV, genus Masterevirus, family Geminiviridae) and is the most important virus disease of maize in Africa [1]. MSV, transmitted by several species of Cicadulina leafhoppers, is endemic in all the maizeproducing countries in Africa and its offshore islands and is not known to occur outside the continent. The infected plants have yellow streaks that run parallel to the leaf veins. The leaves that emerge after MSV infection show symptoms, whereas older leaves remain healthy. Susceptible cultivar develop severe streaking and infection at the early stages of plant growth, leading to severe stunting, interveinal necrosis, and death of affected plants [2]. Yield losses from MSD vary with the time of infection and varietal resistance, reaching $100 \%$ in susceptible lines [3,4]. Annual incidence of MSD in farmers' fields varies from a few infected plants with insignificant yield losses to nearly $100 \%$ infection in others, resulting a very high loss $[5,6]$. On average, MSV could cause annual yield losses in Africa estimated at US\$120-480 million [7].

Host resistance is the most cost-effective option for managing MSD in sub-Saharan Africa. The first reports of MSV resistance were in cultivars Peruvian Yellow and Arkkels 
Hickory, and an incompletely dominant gene was reported in the cross between them [8]. International Institute of Tropical Agriculture (IITA) researchers detected MSV resistance in the Tropical Zea Yellow (TZY) population that has been used as a source of resistance in several breeding programs in Africa [9]. Host resistance to MSD is a recovery type that exhibits reduced severity of symptoms in the leaves emerging after infection with low virus titers and good yields are produced despite infection $[5,10]$.

Breeding programs at IITA and several organizations in the past 50 years have developed and released several MSD-resistant varieties. Resistance to MSD is considered an essential adaptive trait, and breeding programs have been incorporating it continuously in their development of new varieties intended for release in Africa. The conventional system of incorporating resistance involves crossing the best plants with the most desirable traits, such as high yield, disease resistance, and other farmer preferred characters. Screening of early generation lines for recovery resistance to MSV using viruliferous leafhoppers in screenhouse and field may take more than five to seven years to finally develop a variety that has acceptable levels of resistance [9].

Development and use of markers for selection in crop improvement have become a useful technique for breeders. Since the MSD resistance trait has high heritability, controlled by a few genes [11-13], the application of markers in transferring genes for resistance can be easier and quicker than the conventional selection using phenotyping. Molecular markers can help breeders select individuals carrying target genes in a segregating population based on patterns of tightly linked markers with due regard to their phenotypes. The quantitative trait loci (QTLs) for resistance to MSD in maize have been identified and mapped. Kyetere et al. [14] identified a major gene on chromosome 1 (Msv1) in Tzi4, a tolerant line from IITA. Welz et al. [13] detected a major QTL on chromosome 1 and minor QTLs on chromosomes 2,3, and 4. The presence of a major QTL for resistance to MSD on the short arm of chromosome 1 has been identified in diverse germplasm and across environments [11-16]. Nair et al. [17] mapped Msv1 and identified 19 SNPs (single nucleotide polymorphism) in the region between 82 and $93 \mathrm{Mb}$ on chromosome 1. Three SNPs (snpZM0020-PZE101093951, SnpZM0021-PZE-0186065237, and snpZM0021-PZE0186365075) were identified in the haplotype block at $87 \mathrm{Mb}$ on chromosome 1 and found an accuracy of 0.94 in predicting Msv1 QTL in maize lines [17]. High-throughput kompetitive allele specific PCR assays (KASP) have been developed for these three SNPs as potential breeder-ready markers for the indirect selection of MSV resistance in breeding pipelines.

This study was conducted to validate the three SNP markers to predict Msv1 resistance in early generation tropical maize inbred lines with an ultimate objective of confirming whether the breeder-ready markers can be extensively used to select MSV resistance in tropical maize breeding programs in Africa.

\section{Materials and Methods}

\subsection{Genotyping of Early Generation Maize Inbred Lines}

A total of 151 early generation maize lines derived from nine bi-parental crosses were selected from among the $3015 \mathrm{~S} 1$ lines for genotyping for the three SNP markers (snpZM0020-PZE101093951, snpZM0021-PZE0186065237, and snpZM0022-PZE0186365075) linked to the alleles associated with the resistance QTL on chromosome 1 of the B73RefGe$\mathrm{n} \_\mathrm{V} 2$ (Table 1). For genotyping, leaf tissues (two leaf discs of $0.5 \mathrm{~cm}$ diameter) from 151 maize lines were harvested 21 days after planting. These were freeze-dried and shipped to the Intertek laboratory in Sweden for DNA extraction and SNP genotyping using the three SNP markers with the KASP SNPline (LGC Genomics). Genotype allele calling was implemented in Kraken caller software (LGC Group). 
Table 1. Primer sequences of kompetitive allele specific PCR-single nucleotide polymorphism (KASP-SNPs) used in genotyping.

\begin{tabular}{|c|c|c|c|c|}
\hline SNP ID * & Intertek ID & Target Sequence $\left(5^{\prime} \rightarrow 3^{\prime}\right)$ & $\begin{array}{c}\text { MSV Resistance } \\
\text { Allele }\end{array}$ & $\begin{array}{c}\text { Susceptible } \\
\text { Allele }\end{array}$ \\
\hline PZE-101093951 & snpZM-0020 & $\begin{array}{c}\text { TAACTCTCTGCTGTTGCTTGTCTTCAG } \\
\text { GTTGTCATGAGAGATCCTCACAT[A/G] } \\
\text { GCAGCAGATGGCTTCACCTACGAAG } \\
\text { CTGACGCTCTTAGATACTGGCTCGA } \\
\text { ACATCTCCAGTAACAA ACAGAAGTC }\end{array}$ & A:A & $\mathrm{G}: \mathrm{G}$ \\
\hline PZE-0186065237 & snpZM-0021 & $\begin{array}{c}\text { TTTCGAATCGTGATACCATCCCCA } \\
\text { A[T/C]CACGCACTGCGKTCGGCC } \\
\text { ATCCAAGAATACCTCCGGCAGAAC } \\
\text { GAGCTGCA }\end{array}$ & $\mathrm{C}: \mathrm{C}$ & $\mathrm{T}: \mathrm{T}$ \\
\hline PZE-0186365075 & snpZM-0022 & $\begin{array}{c}\text { AGAAGAAAATGGCCTGCCATATATAT } \\
\text { ATCCCGGTTAATCGCTARTGCATT[A/C] } \\
\text { TCAGGAATCATTCTCATAGGTCATA } \\
\text { AGACGAGCAAGGGATACTCTTCTAC }\end{array}$ & $C: C$ & A:A \\
\hline
\end{tabular}

\subsection{Phenotyping for MSV Response}

The 151 genotyped lines and 9 elite lines with varying levels of field resistance to MSV were phenotyped under screenhouse conditions at IITA, Ibadan station in Nigeria (190 masl; latitude $7^{\circ} 29^{\prime} 11.99^{\prime \prime} \mathrm{N}$, longitude $3^{\circ} 54^{\prime} 2.88^{\prime \prime} \mathrm{E}$ ). Seed lots of the 160 test lines and Pool-16, that was used as MSV susceptible check, were collected from IITA Maize Breeding Unit at Ibadan Station and planted for MSV resistance response in an insectproof screen house under natural conditions (mean temperature between 21.4 and $26.5^{\circ} \mathrm{C}$, mean relative humidity of $74.6 \%$ ) as outlined by Soto et al. (1982). The MSV isolate and leafhopper colonies (C. triangular) used in this study were maintained at the IITA Virology and Molecular Diagnostic Unit at Ibadan. Non-viruliferous insect colonies were reared and maintained on seedlings of pearl millet (Pennisetum typhoides) maintained in insect-proof cages. After giving $48 \mathrm{~h}$ of acquisition access period (AAP) on MSV-infected Pool-16 in insect-proof cages, young adults were used for MSV transmission as follows.

The 160 maize lines were arranged in a completely randomized design with two replications. Each line was considered a treatment. For each rep, eight seeds of each line and four seeds of the susceptible control Pool-16) were sown per pot and after one-week ( 2 to 3-leaf stage) pots were placed in cages for virus inoculation feeding, and the viruliferous leafhoppers were allowed a 48 -h inoculation access period (IAP). This procedure allowed uniform inoculation of all lines; Pool-16 was planted in each pot as control for assessing inoculation efficiency. Following inoculation, plants were removed from the cages and sprayed with lambda-cyhalothrin insecticide (Karate 5 EC, Syngenta, Switzerland) at $60 \mathrm{~mL} / 20 \mathrm{~L}$ water to kill vectors. The plants were transferred to an insect-proof screenhouse for observation.

MSV incidence and severity were assessed one-week post inoculation (wpi) as detailed in Soto et al. (1982). Disease severity on newly formed leaves of each test plant was assessed at weekly intervals for 6-wpi using a 1 to 5 symptom severity rating scale where $0=$ no symptoms, $1=10 \%$ of the leaf area covered with streaks, $2=11-25 \%$ covered, $3=26-50 \%$ covered, $4=51-75 \%$ covered, and $5 \geq 75 \%$ covered with streaks. The symptom severity scores of each test line at 6 wpi were used to define resistance categories as follows: 0.1 to 1 highly resistant (HR), 1.0 to 2.0 resistant (R), 2.1 to 3.0 moderately resistant (MR), 3.1 to 4.0 susceptible (S), and 4.1 to 5.0 highly susceptible (HS).

\subsection{Determination of Relative Accumulation of MSV by Enzyme-Linked Immunosorbent Assay (ELISA)}

Previous studies have established a positive relationship between the severity of streak disease symptoms and MSV titer in the maize leaves [18]. The direct antigen coating (DAC)- 
ELISA format as described by Peterschmitt et al. [18] was used to monitor the accumulation of MSV with the most symptomatic leaves (at the bottom of each test line) and the leastsymptomatic (top leaves) as a measure of recovery response using MSV rabbit polyclonal antibodies produced against purified MSV virions (Virology and Molecular Diagnostics Unit, IITA, Nigeria). At 6 wpi, leaf discs of $1 \mathrm{~cm}$ diameter $(\approx 50 \mathrm{mg})$ were harvested from the bottom leaf (the first leaf to emerge after leafhopper inoculation) and the fully expanded top leaf for separate testing. Two plants/replication/line were sampled, and samples of the same leaf stage for each replication were pooled (2 composite samples/leaf stage/line) for testing in ELISA.

The leaf discs were homogenized in $0.5 \mathrm{~mL}$ antigen coating buffer $\left(15 \mathrm{mM} \mathrm{Na}_{2} \mathrm{CO}_{3}\right.$, $35 \mathrm{mM} \mathrm{NaHCO}_{3}, \mathrm{pH} 9.6$ ) and $100 \mu \mathrm{L}$ of homogenate was added into a 96-well flat-bottomed polystyrene microtiter plate (Corning CLS9017, Sigma-Aldrich, St. Louis, MO, USA) and incubated overnight at $4{ }^{\circ} \mathrm{C}$. In each ELISA plate, virus positive control was MSV-infected Pool-16, uninfected maize was healthy control, and coating buffer was no template control. All samples were tested in duplicate wells. The MSV antigen and the antibody reactions were detected using alkaline phosphatase conjugated goat anti-rabbit antiserum (Cat. No. A3687-1ML, Sigma-Aldrich, St. Louis, MO, USA) and p-nitrophenyl phosphate (SigmaAldrich, St. Louis, MO, USA) substrate. The ELISA plates were incubated in the dark at room temperature for two hours. Absorbance was measured at $405 \mathrm{~nm}$ (A405 nm) using an ELISA plate reader (New Micro Read 1000, Global Diagnostics, Belgium). Wells with absorbance at $\mathrm{A} 405 \mathrm{~nm}$ readings twice that of the healthy control were considered positive to MSV. Leaf samples from all test plants were sampled at 6 wpi to evaluate the relative accumulation of the virus in the leaf extract based on the A405 nm values that were used to estimate absorbance fold value (AFV) (ratio) compared with the healthy control using the formula below.

$$
A F V=\frac{A 405 \mathrm{~nm} \text { of test leaf }}{2 x(\text { A405nm of healthy control })}
$$

The AFV values were used as a proxy to determine the level of MSV accumulation in the leaves of the test plants. Similarly, the difference in MSV accumulation as absorbance fold difference (AFD) between the bottom and top leaves was calculated using the formula below.

$$
A F D=\frac{A F V \text { of bottom leaves }}{A F V \text { of top leaves }}
$$

\subsection{Data Analysis}

The percentage symptom recovery was calculated from formula given below, based on the percentage reduction in symptom severity scores between the first leaf after inoculation ( 2 to $3 \mathrm{wpi}$ ) and the newly emerged leaf at the last observation (6 wpi).

Percentage recovery $=100-(($ Minimum score value $) /($ Maximum score value $)) \times 100$

where minimum score value is the mean symptom severity score of a top leaf (6-wpi); and maximum score value is the mean symptom severity score of the first emerged leaf ( 2 or 3 wpi) after inoculation.

The data on symptom severity of leaves of inoculated plants and ELISA were subjected to analyses of variance using PROC GLM in SAS program version 9.4 (SAS Institute Inc., Cary, NC, USA). Chi-square test was performed to test the association between SNPs and the mean symptom severity score. Proc GLM was used to assess whether there was a significant mean symptom score among the maize lines and Pearson correlation analysis was performed to determine the relationship between mean symptom score and the A405nm absorbance values of ELISA. All correlation analyses were carried out using PROC CORR in SAS. The area under the disease progress curve (AUDPC) was calculated for the disease severity scores using the formula of Wilcoxson et al. [19] as follows:

$$
\operatorname{AUDPC}=\Sigma\left(\left(\mathrm{x} \__{-} \mathrm{i}+\mathrm{X}_{-}(\mathrm{I}+1)\right) / 2\right) \cdot\left(\mathrm{t} \_(\mathrm{i}+1)-\mathrm{t} \_\mathrm{i}\right)
$$


where $x i$ is the disease rating on date $i$ and $t i$ is the time (in calendar days) on which $x i$ is recorded; $\mathrm{I}=1,2 \ldots 5$.

\section{Results}

\subsection{MSV Symptom Severity Scores and Relationship with Alleles of SNP Markers}

Among the 151 lines genotyped in this study, 133 had the three SNP markers associated with MSV-resistant alleles of Msv1 and the remaining 18 lines did not (Supplementary Table S1). Inoculation using viruliferous leafhoppers resulted in $100 \%$ transmission of MSV in the susceptible control (Pool-16) and test lines. All infected plants developed sharp, discontinuous chlorotic streaks within 3 to 4 days after inoculation (Figure 1). The virus incidence on 160 test lines and the susceptible check was 100\% at the end of the first week after inoculation (wpi), indicating uniformity of leafhopper inoculation. Most severe symptoms were observed in the leaves that emerged after inoculation. In all the lines with MSV-resistant alleles and the nine elite lines, the first emerged leaves after inoculation developed severe symptoms between 1 and $2 \mathrm{wpi}$, and symptoms were reduced by 6 wpi (Figure 1). The mean symptom severity scores for the 133 test lines with Msv1 alleles were the highest at 2 wpi $(3.9 \pm 0.5)$ and declined to $2.2 \pm 0.7$ at $6 \mathrm{wpi}$, with an average of $3.1 \pm 0.4$ across the weeks (Figure $2 \mathrm{~A}$ ). The nine elite lines had the highest score of $4.3 \pm 0.5$ and declined to $1.7 \pm 0.8$ ) at $6 \mathrm{wpi}$, with an average of $3.0 \pm 0.7$ across the weeks (Figure 2C). In contrast, severity of streak symptoms increased in lines without SNPs for MSV resistance up to the 3-wpi and had only a slight remission up to 6 wpi, with an average of $4.0( \pm 0.4)$ across the weeks (Figure 2B, Supplementary Table S1, and Table 2).
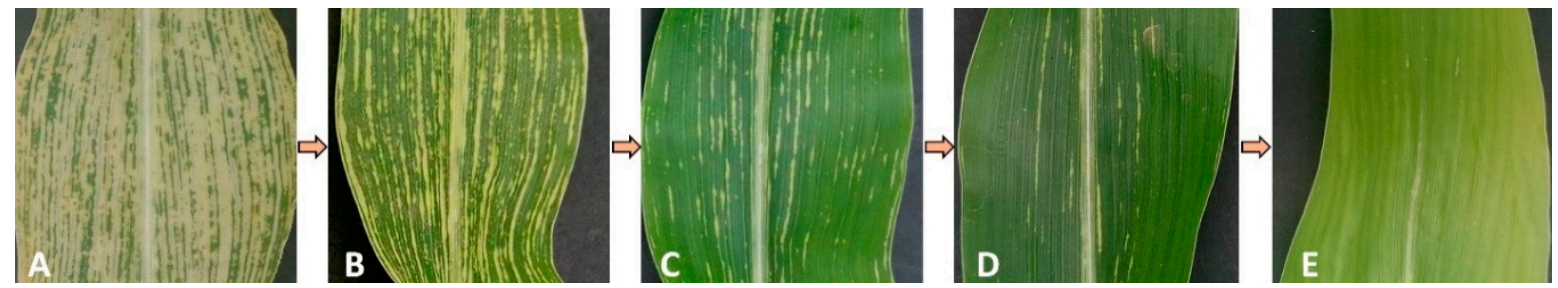

Figure 1. Reduction in symptom severity in the newly emerging leaves of a maize line with recovery resistance to maize streak virus (MSV) infection. Left to right: the first leaf emerged after inoculation showing severe symptoms (A) and a gradual reduction in severity in subsequently emerged leaves (B-E).

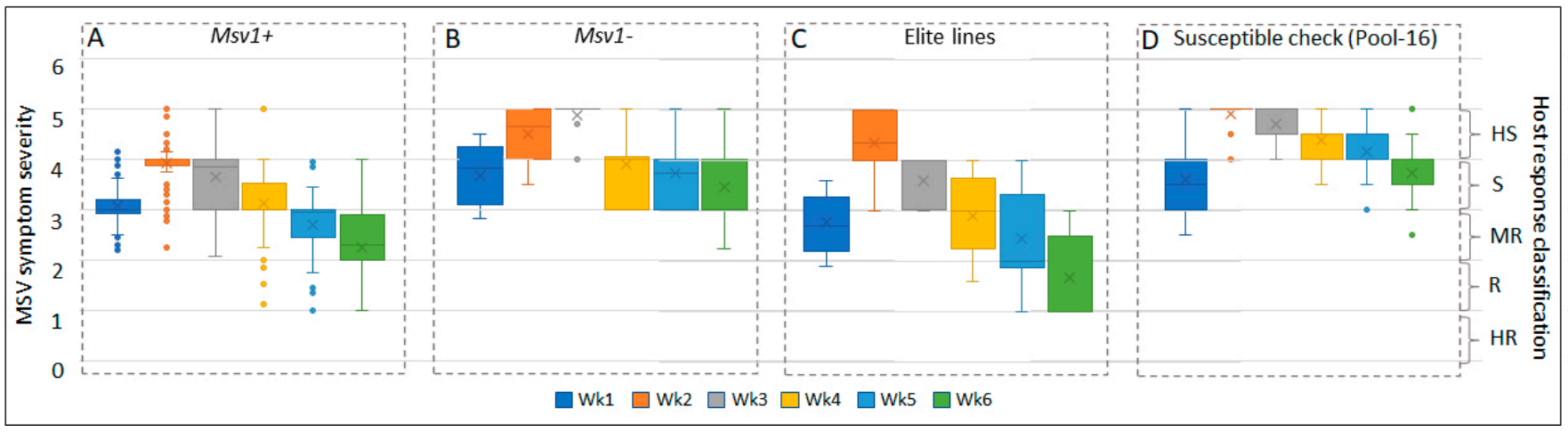

Figure 2. Box plots demonstrating maize streak virus (MSV) symptom severity (y-axis) from 1 to 6 wpi of seven maize bi-parental lines with the MSV resistant allele (Msv1+) (A), three bi-parental lines without the MSV resistant allele (Msv1-) (B), nine MSV resistant elite lines (C), and the susceptible check, cv. Pool-16 (D). Host resistance classification based on symptom severity is indicated as $\mathrm{HR}=$ highly resistant ( 0.1 to 1.0$), \mathrm{R}=$ resistant (1 to 2$), \mathrm{MR}=$ moderately resistant (2.1 to 3.0), $\mathrm{S}=$ susceptible (3.1 to 4.0 ), and HS = highly susceptible (4.1 to 5.0). 
Table 2. Summary of mean symptom severity score and relative MSV load in maize bi-parental crosses and inbred lines.

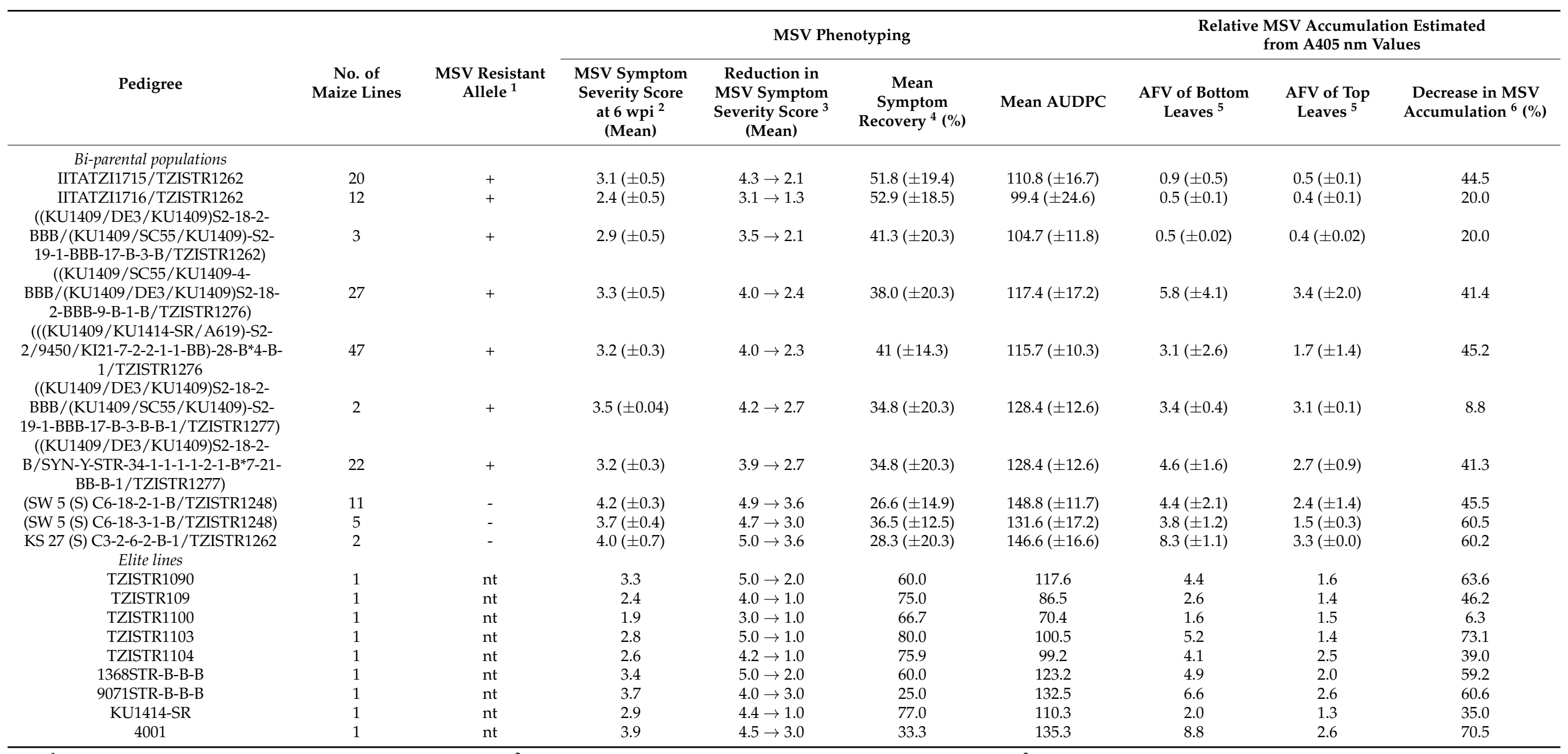

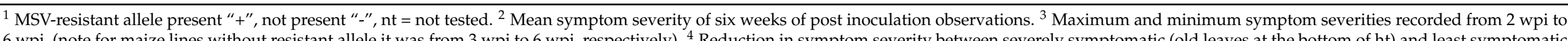

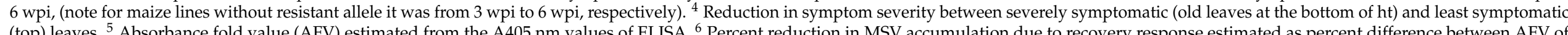

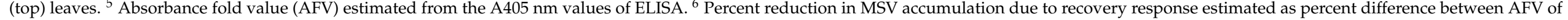
bottom (most severe symptoms) and top leaves (least symptoms). 
Of the 1221 Pool-16 plants inoculated, $93 \%$ had mean symptoms score between 4.1 and 5.0 (HS), and 6.9\% had a mean severity score between 3.1 and 4.0 (S) (Figure 2D). This distribution of symptom severity in Pool-16 was within the expected range for a susceptible genotype and indicated the effectiveness of the MSV phenotyping procedure. The analyses of variance for the mean MSV symptom score were highly significant. All the factors, viz., lines, scoring weeks, and lines $\times$ weeks differed significantly $(p<0.01)$ for the mean symptom score, suggesting the differential response of lines for MSV over the week. The mean square for scoring weeks was much higher (149.58) than those of the lines (3.58) and the line $\times$ scoring week interaction (0.34), indicating the rapid changes in scores over time. The severity of streak symptoms declined from the lower (older) leaves to the upper (newer) leaves as expected for the recovery type of resistance. Most of the lines had symptom score value of 5 at the older leaves and declined up to score value 1 on upper, newly emerged leaves depending on their recovery resistance. Significant differences $(p<0.01)$ in MSV symptom severity was obtained among the 160 maize lines at six weeks after inoculation with LSD (0.05) value of 0.19 and coefficient variation of 5.4.

Out of the 160 lines assayed based on mean symptom scores recorded at $6 \mathrm{wpi}, 37$ lines had scores varying from 1.0 to 2.0 (R), 88 had scores varying from 2.1 to 3.0 (MR), 8 were rated from 3.1 to $4.0(\mathrm{~S})$, and 3 had scores of 4.1 to 5.0 (HS) (Table 3; Figure 3). Among the 133 maize lines having the three alleles associated with MSV resistance and the 9 elite lines, 54 had MSV symptom scores ranging from 1.0 to 2.0 (R), 76 had scores of 2.1 to 3.0 (MR), and 3 had scores varying from 3.1 to 4.0 (S) (Tables 2 and 3). None of the 18 maize lines without alleles for resistance to MSV had symptom scores in the resistance range (1.0-2.0). However, 10 of these lines had MSV symptom scores varying from 2.1 to 3.0 (MR), 5 had scores of 3.1 to 4.0 (S), and 3 had scores of 4.1 to 5.0 (HS) (Tables 2 and 3). Of the 9 elite lines tested, 7 had symptoms scores in the resistance range (1.0-2.0), and 2 had MSV symptom scores between 2.1 and 3.0 (MR) (Tables 2 and 3).

Table 3. Distribution of maize lines with and without Msv1 alleles based on the MSV symptom severity score at 6 wpi.

\begin{tabular}{|c|c|c|c|c|c|}
\hline \multirow{2}{*}{ Type } & \multicolumn{4}{|c|}{ Severity Rating } & \multirow[b]{2}{*}{ Total } \\
\hline & $1.0-2.0(\mathrm{R})$ & $2.1-3.0$ (MR) & $3.1-4.0(\mathrm{~S})$ & $4.1-5.0$ (HS) & \\
\hline $\begin{array}{l}\text { Early generation lines with three SNPs } \\
\text { associated with MSV resistance }\end{array}$ & 54 & 76 & 3 & 0 & 133 \\
\hline $\begin{array}{l}\text { Early generation lines without SNPs for } \\
\text { MSV resistance }\end{array}$ & 0 & 10 & 5 & 3 & 18 \\
\hline Elite maize lines & 7 & 2 & 0 & 0 & 9 \\
\hline Total & 61 & 88 & 8 & 3 & \\
\hline
\end{tabular}

$\mathrm{R}=$ resistant $\mathrm{MR}=$ moderately resistant $\mathrm{S}=$ susceptible, $\mathrm{HS}=$ highly susceptible.

A Chi-square value $\left(\chi^{2}=52.25, \mathrm{df}=3\right)$ obtained from the test of association between alleles of the SNP markers and MSV symptoms was significant $(p<0.01)$, indicating that the marker allele was linked with MSV resistance in the tested lines. Of the 133, maize lines with the 3 SNPs associated with MSV resistance alleles, 130 were $\mathrm{R}(n=54)$ or MR $(n=76)$, showing a success rate of $98 \%$ in identifying resistant lines. However, 3 lines (2.2\%) with the Msv1 resistant allele were susceptible, whereas 10 (55\%) that did not carry the resistance allele for MSV resistance were found to be moderately resistant. This could arise from the inability of the SNPs to consistently detect and identify resistance alleles or from the presence of other QTLs contributing to the MSV resistance response [15,20].

Percentage recovery varied from 0 to $80 \%$ for the lines tested (Table 2). In lines with MSV-resistant alleles, the mean symptom severity went down from $3.9 \pm 0.6$ at 2 wpi to $2.2 \pm 0.7$ at 6 wpi (Table 2). Similarly, the symptom recovery in maize lines without SNPs for MSV resistance declined from $4.9 \pm 0.2$ at 2 wpi to $3.4 \pm 0.3$ at 6 wpi. Of the 160 lines, 58 had $>50 \%$ recovery, whereas 102 had $<50 \%$ recovery. Out of the 58 lines with a high percentage recovery, only one line lacked the SNP alleles for MSV resistance. High rates of recovery (33 to $77 \%$ ) were observed in $43 \%$ of the lines that were positive for 3 SNP markers 
associated with MSV resistance; whereas 55\% showed a moderate recovery varying from 24 to $53 \%$; the remaining $2 \%$ showed low recovery of 5 to $23 \%$. There were marked differences among the lines for AUDPC values (Table 2).

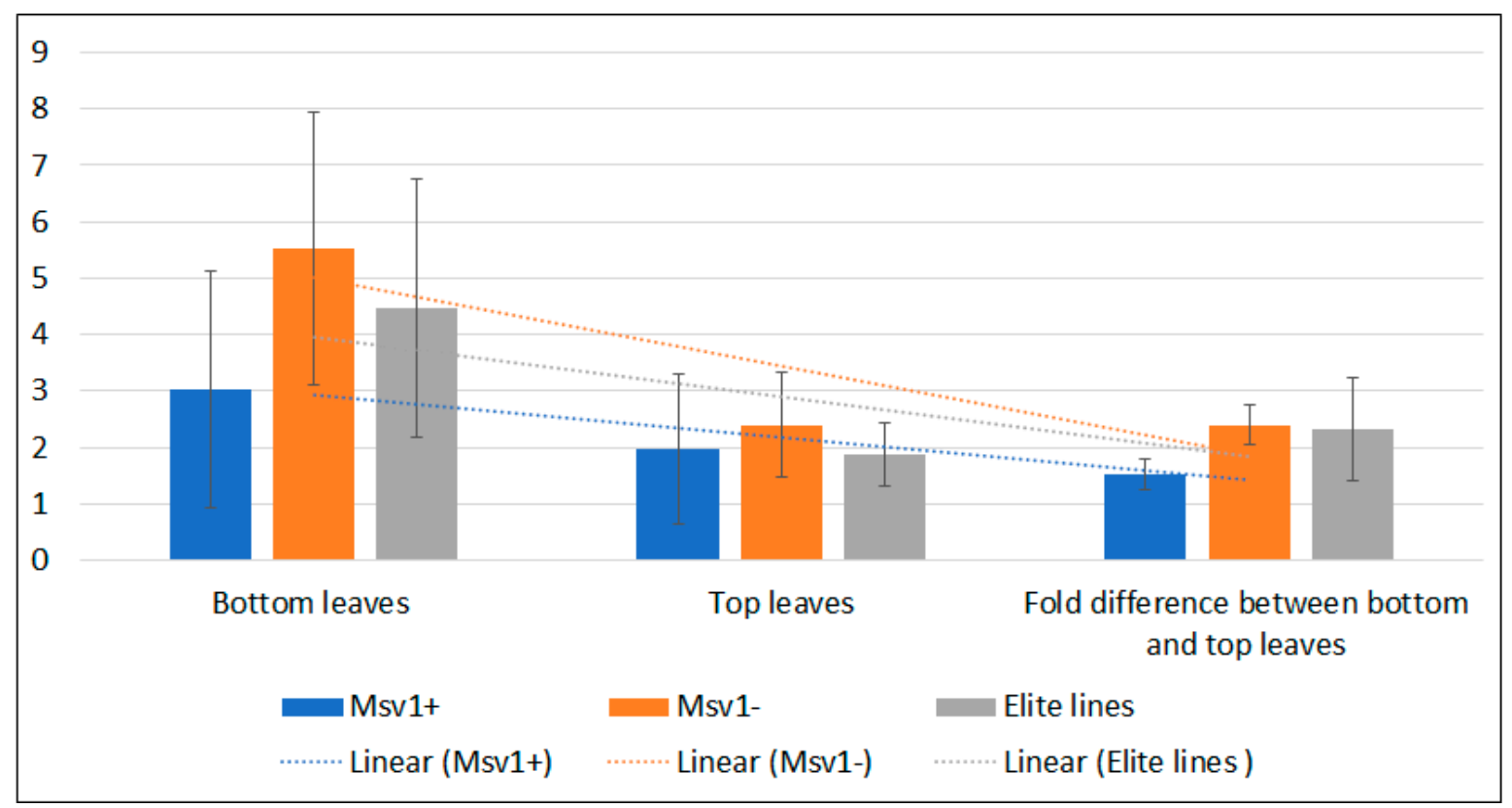

Figure 3. Mean relative MSV load (y-axis) (estimated by dividing the A405 nm values of test leaves by $2 \times$ A 405 value of health leaf control) in maize bi-parental crosses with (+) and without (-) MSv1 resistant allele and nine elite lines. Trendlines indicate a general pattern of reduction in MSV accumulation between maize lines with/without Msv1 allele and a decrease in virus load in top leaves (recovered leaves) compared with bottom leaves.

\subsection{Variation in Recovery Response among Lines Derived from Biparental Crosses}

The lines derived from each of the 10 bi-parental crosses showed scores varying from 2 to 47 (Table 2). The lowest mean MSV severity score was observed for lines developed from IITATZI1715/TZISTR1262 and ITATZI1716/TZISTR1262, followed by lines derived from ((KU1409/DE3/KU1409)S2-18-2-BBB/(KU1409/SC55/KU1409)-S2-19-1-BBB-17-B-3B/TZISTR1262). About 54\% of the lines derived from IITATZi1715/TZiSTR1262 showed the best recovery resistance compared with lines derived from other crosses (Table 2). Twenty out of the thirty-two lines derived from IITATZi1715/TZiSTR1262 had symptom severity scores lower than or equal to 2 . In resistant lines, the symptoms gradually decreased from the lower to the upper leaves. Amongst the elite maize inbred lines, TZiSTR1100 had the lowest MSV symptom score of 1.9. Lines developed from three bi-parental crosses (SW5(S)C6-18-2-1-B/TZiSTR1248, KS27(S)C3-2-6-2-B-1/TZiSTR1262, and SW5(S)C6-183-1-B/TZISTR1248) without the MSV resistant allele had the highest average score of symptom severity, indicating high susceptibility to MSV. Nine of the ten maize lines without resistant alleles showed MSV symptom scores of 2-3 (MR) deriving from SW5(S)C6-183-1-B/TZISTR1248. These results suggest that additional QTLs probably controlled the resistance in these lines.

\subsection{MSV Detection and Relative Titer Estimation Using ELISA}

The A405 $\mathrm{nm}$ values of the test lines results were converted into ratios against a baseline ( $2 \times$ A405 nm of health control) and were presented as MSV load (Figure 3). The mean MSV load in severely infected bottom leaves was $3.4 \pm 3.0$ and in most recovered leaves at the top of the lines with MSV resistant alleles it was $1.9 \pm 1.6$; whereas the virus load was $4.7 \pm 2.2$ in the bottom leaves of the lines without resistant SNP alleles and $2.2 \pm 1.2$ in the top leaves (Table 2). The estimated MSV load values ranged from 0.3 
to 13.4 for the bottom leaves and from 0.3 to 8.5 for the recovered leaves in lines with MSV-resistant alleles. The MSV was undetectable in both upper and bottom leaves in 47 lines, whereas it was detected at low levels in lower leaves but not in the upper leaves in 14 lines (Supplementary Table S1). In general, the ELISA data indicated a decrease in MSV accumulation in the upper leaves rather than in the leaves that emerged immediately after inoculation. For both the lower and the upper leaves, the association between MSV titer response and the SNP markers allele was significant $(p<0.01)$ for upper $\left(\chi^{2}=78.63\right.$, $\mathrm{df}=1)$ and lower $\left(\chi^{2}=13.6, \mathrm{df}=1\right)$ leaves. Chi-square analysis of the relationship between the resistant/susceptible class (resistant, moderate resistant, and susceptible) and MSV load status showed significant $(p<0.01)$ association for both the lower $(\chi 2=17.62$, $\mathrm{df}=2)$ and upper $\left(\chi^{2}=38.75, \mathrm{df}=2\right)$ leaves. There was a significant association at six weeks after inoculation between the presence or absence of SNP alleles in the lines and MSV accumulation.

Pearson correlation analysis were carried out between the mean symptom score and ELISA result. There was a significant correlation among the result obtained from the mean severity score and ELISA result. The mean symptom severity score value had a strong positive correlation $(p<0.01)$ with AUDPC and the virus titer on the upper and lower leaves with a correlation coefficient value of $0.96,0.29$, and 0.31 , respectively. The virus titer on the upper and lower leaves had a strong positive correlation $(p<0.01)$ with a correlation coefficient value of 0.89 .

\section{Discussion}

MSV is one of the most economically important diseases of maize in Africa. Host resistance is considered to be the most practical, cost-effective, and environmentally acceptable means for managing MSV and other foliar diseases in sub-Saharan Africa [21]. Historically, resistance breeding in maize for foliar diseases has focused on selecting a high level of quantitative resistance to safeguard against the potential evolution of new strains of the pathogen that will overcome resistance without significant yield losses [1,22]. Quantitative resistance to MSV identified in a few maize lines was found to be governed by many loci $[5,15,17]$. Using DNA markers, a major locus (Msv1) mapped to chromosome 1 that accounted for between 40 and $76 \%$ of phenotypic variation was found [11,12,14], and three SNPs were identified to have a Msv1 resistant allele prediction efficiency of 0.94 [17]. The prediction potential of the three SNP markers Msv1 was validated in this study by analyzing 151 early generation lines with and without MSV-resistant SNP alleles along with nine MSV resistant elite lines as a reference set.

The MSV inoculation procedure using leafhopper vectors was robust as no plant, including the susceptible reference genotype, escaped infection among the test lines. The inoculated plants developed first symptoms $3-5$ days after inoculation. The symptom severity gradually decreased from the lower to the upper leaves due to the type of resistance that is referred to as recovery resistance to MSV in maize [10,23]. The symptom severity increased to maximum level within $2 \mathrm{wpi}$, and then declined to the lowest level at $6 \mathrm{wpi}$ in the 133 lines carrying alleles for Msv1 and in the nine elite lines used as reference sets. In the 18 maize lines without the resistant allele, symptom severity peaked at 3 wpi and was lowest at 6 wpi. Among lines with the SNPs associated with MSV resistance, $41 \%$ showed the highest rate of recovery, $57 \%$ had a moderate recovery, and about $2 \%$ had none.

The ELISA detection method further confirmed the results obtained from phenotyping following leafhopper inoculation. The virus titer of the leaf sample is a function of the level of resistance/susceptibility reactions of the maize line, the time after infection when leaves were assayed, and the position (top or bottom) on the leaf assayed [18]. Samples from the severely symptomatic bottom leaves harvested from 2 to 3 wpi were associated with high virus titer and those from the least symptomatic top leaves harvested at 6 wpi were associated with low virus titer. The alleles of the SNP markers were strongly associated with the leaf virus titer response of the upper leaf, consistent with the results obtained for recovery resistance to MSV of the lines used in the current study. 
The three SNP markers in the present study identified 130 of the 133 maize lines as resistant or moderately resistant lines. The phenotyping in addition delineated about $50 \%$ of 18 maize lines without Msv1 QTLs as moderately resistant to MSV which suggests the involvement of additional QTLs that control MSV resistance in the test lines. These results indicated that the three SNP markers were useful for identifying MSV resistance in many resistant lines. However, additional markers are needed to identify lines with clearly defined resistance categories. In another study, 18 QTLs mapped on eleven genomic regions covering chromosomes 1, 2, 3, 45, and 7 associated with different components of MSV resistance accounted for $3.1-21.4 \%$ of phenotyping variance for MSV [15]. The presence of other resistance QTLs on chromosome 7 highlights the need for fine mapping of other QTLs to identify additional potential SNP markers for breeding [15].

MAS is cost-effective and offers efficiency in phenotyping for MSV [24]. The three SNP diagnostic markers used in this study are suitable for the screening of early-generation lines for resistance to MSV, which is essential for nomination of an improved line in the varietal release pipeline in Africa. However, additional markers are required to identify other QTLs responsible for accurately differentiating inbred lines into clearly defined categories of MSV resistance to save breeding costs for identifying superior inbred lines.

Supplementary Materials: The following are available online at https:/ /www.mdpi.com/2077-0 472/11/2/130/s1, Table S1: Summary of maize streak virus phenotyping, genotyping, ELISA data, phenotypic and ELISA test results.

Author Contributions: Conceptualization, P.L.K., A.M., and M.G.; methodology, P.L.K., A.M. and M.G.; formal analysis, S.S.S., P.L.K., and A.M.; investigation, S.S.S., P.L.K., A.M. and M.G.; resources A.M., P.L.K. and V.O.A.; data curation, S.S.S., P.L.K. and A.M.; writing-original draft preparation, S.S.S. and P.L.K.; writing-review and editing, S.S.S., P.L.K., A.M., M.G. and V.O.A.; supervision, P.L.K., A.M. and V.O.A.; funding acquisition, A.M., P.L.K. and M.G. All authors have read and agreed to the published version of the manuscript.

Funding: This research was funded by the CGIAR Research Program on MAIZE (CRP MAIZE), and the project (OPP1134248) on Stress Tolerant Maize for Africa (STMA) funded by Bill \& Melinda Gates Foundation (BMGF). The first author is grateful to the African Union (AU) for providing a fellowship for MSc study at the Pan African University Earth and Life Sciences at the University of Ibadan, Nigeria.

Institutional Review Board Statement: Not applicable.

Informed Consent Statement: Not applicable.

Data Availability Statement: Provided in Supplementary Table S1.

Acknowledgments: Authors acknowledge the support of IITA staff members, A. Oyelakin for assistance with rearing of leafhoppers and maintenance of experimental plants under screenhouse conditions, P. Ogunsanya for assistance with ELISA, and O. Ilesanmi for preparing maize samples for KASP assay. We thank the BMGF (project \#OPP1134248) for supporting open access publishing fees.

Conflicts of Interest: The authors declare no conflict of interest. The funders had no role in the design of the study; in the collection, analyses, or interpretation of data; in the writing of the manuscript, or in the decision to publish the results.

\section{References}

1. Pratt, R.C.; Gordon, S.G. Breeding for resistance to maize foliar pathogens. Plant Breed. Rev. 2006, 27, 119-174.

2. Thottappilly, G.; Bosque-Perez, N.A.; Rossel, H.W. Viruses and virus diseases of maize in tropical Africa. Plant Pathol. 1993, 42, 494-509. [CrossRef]

3. Guthrie, E.J. Measurement of yield losses caused by maize streak disease. Plant Dis. 1978, 62, 839-841.

4. Magenya, O.E.V.; Mueke, J.; Omwega, C. Significance and transmission of maize streak virus disease in Africa and options for management. Afr. J. Biotechnol. 2008, 7, 4897-4910.

5. Efron, Y.; Kim, S.K.; Fajemisin, J.M.; Mareck, J.H.; Tang, C.Y.; Dabrowski, Z.T.; Rossel, H.W.; Thottappilly, G.; Buddenhagen, I.W. Breeding for resistance to maize streak virus: A multidisciplinary team approach. Plant Breed. 1989, 103, 1-36. [CrossRef] 
6. Shepherd, D.N.; Martin, D.P.; van de Walt, E.; Varsani, K.D.A.; Rybicki, E.P. Maize streak virus: An old and complex 'emerging' pathogen. Mol. Plant Pathol. 2010, 11, 1-12. [CrossRef] [PubMed]

7. Martin, D.P.; Shepherd, D.M. The epidemiology, economic impact and control of maize streak disease. Food Secur. 2009, 1, 305-315. [CrossRef]

8. Storey, H.H.; Howland, A.K. Inheritance of resistance in maize to the virus of streak disease in East Africa. Ann. Appl. Biol. 1967, 59, 429-436. [CrossRef]

9. Soto, P.E.; Buddenhagen, I.W.; Asnani, V.L. Development of streak virus-resistant maize populations through improved and selection methods. Ann. Appl. Biol. 1982, 100, 539-546. [CrossRef]

10. Bosque-Perez, N.A. Eight decades of maize streak virus research. Virus Res. 2000, 71, 107-121.

11. Pernet, A.D.; Hoisington, J.; Dintinger, D.; Jewel, C.; Jiang, C.; Khairallah, M.; Letourmy, P.; Marchand, J.L.; Glaszmann, J.C.; de leon, D.G. Genetic mapping of Maize streak virus resistance from the Mascarene source II. Resistance in line CIRAD390 and stability against across germplasm. Theor. Appl. Genet. 1999, 99, 540-553. [CrossRef]

12. Pernet, A.D.; Hoisington, J.; Franco, M.; Isnard, M.; Jewel, C.; Jiang, C.; Marchand, J.L.; Reynaud, B.; Glaszmann, J.C.; de leon, D.G. Genetic mapping of Maize streak virus resistance from the Mascarene source I. Resistance in line D211 and stability against different virus clones. Theor. Appl. Genet. 1999, 99, 524-539. [CrossRef] [PubMed]

13. Welz, H.G.; Schechert, A.; Pernet, A.; Pixley, K.V.; Geiger, H.H. A gene for resistance to the maize streak virus in the African CIMMYT maize inbred line CML 202. Mol. Breed. 1998, 4, 147-154. [CrossRef]

14. Kyetere, D.T.; Ming, R.; McMullen, M.D.; Pratt, R.C.; Brewbaker, J.; Musket, T. Genetic analysis of tolerance to maize streak virus in maize. Genome 1999, 42, 20-26. [CrossRef]

15. Garcia-Oliveira, A.L.; Menkir, A.; Kumar, P.L.; Azuh, V.; Oyetunji, O.J.; Gedil, M. Mapping of new loci for maize streak virus disease resistance in F2:3 population of tropical maize. Cereal Res. Commun. 2020, 48, 195-202. [CrossRef]

16. Lagat, M.; Danson, M.; Kimani, M.; Kuria, A. Quantitative trait loci for resistance to maize streak virus in maize lines used in hybrid development. Afr. J. Biotechnol. 2008, 7, 2573-2577.

17. Nair, S.K.; Raman, B.; Magorokosho, C.; Mahuku, G.; Semagn, K.; Beyene, Y.; Das, B.; Makumbi, D.; Kumar, P.L.; Olsen, M.; et al. Fine mapping of Msv1, a major QTL for resistance to maize streak virus leads to development of production markers for breeding pipelines. Theor. Appl. Genet. 2015, 128, 1839-1854. [CrossRef]

18. Peterschmitt, M.; Reynaud, B.; Sommermeyer, G.; Baudin, P. Characterization of maize streak virus isolates using monoclonal and polyclonal antibodies and by transmission to a few hosts. Plant Dis. 1991, 75, 27-32. [CrossRef]

19. Wilcoxson, R.D.; Skovmand, B.; Latif, A.H. Evaluation of wheat cultivars for ability to retard development of stem rust. Ann. Appl. Biol. 1975, 80, 275-281. [CrossRef]

20. Ladejobi, O.; Salaudeen, M.T.; Kumar, P.L.; Menkir, A.; Adesoye, A.; Atiri, G.; Gedil, M. Mapping of QTLs associated with recovery resistance to streak virus disease in maize. Ann. Agric. Sci. 2018, 63, 115-121. [CrossRef]

21. Shiferaw, B.; Boddupalli, M.P.; Hellin, J.; Bänziger, M. Crops that feed the world 6 Past successes and future challenges to the role played by maize in global food security. Food Secur. 2011, 3, 307-327. [CrossRef]

22. Parlevliet, J.E. Durability of resistance against fungal, bacterial and viral pathogens; present situation. Euphytica 2002, 124, 147-156. [CrossRef]

23. Mesfin, T.; Den Hollander, J.; Markham, P.G. Feeding activities of Cicadulina mbila (Hemiptera: Cicadellidae) on different host-plants. Bull. Entomol. Res. 1995, 85, 387-396. [CrossRef]

24. Abalo, G.; Edema, R.; Derera, J.; Tongoona, P. A comparative analysis of conventional and marker-assisted selection methods in breeding maize streak virus resistance in maize. Crop Sci. 2009, 49, 509-520. [CrossRef] 